\title{
ARTICLE
}

Received 9 Jul 2014 | Accepted 19 Nov 2014 | Published 19 Dec 2014

DOI: $10.1038 /$ ncomms6911

OPEN

\section{Modulator-free quadrature amplitude modulation signal synthesis}

Zhixin Liu', Joseph Kakande ${ }^{2}$, Brian Kelly ${ }^{3}$, John $\mathrm{O}^{\prime}$ Carroll$^{3}$, Richard Phelan ${ }^{3}$, David J. Richardson ${ }^{1}$ \& Radan Slavík ${ }^{1}$

The ability to generate high-speed on-off-keyed telecommunication signals by directly modulating a semiconductor laser's drive current was one of the most exciting prospective applications of the nascent field of laser technology throughout the 1960s. Three decades of progress led to the commercialization of $2.5 \mathrm{Gbits}^{-1}$-per-channel submarine fibre optic systems that drove the growth of the internet as a global phenomenon. However, the detrimental frequency chirp associated with direct modulation forced industry to use external electro-optic modulators to deliver the next generation of on-off-keyed 10 Gbit s $^{-1}$ systems and is absolutely prohibitive for today's $(>) 100 \mathrm{Gbits}^{-1}$ coherent systems, which use complex modulation formats (for example, quadrature amplitude modulation). Here we use optical injection locking of directly modulated semiconductor lasers to generate complex modulation format signals showing distinct advantages over current and other currently researched solutions.

\footnotetext{
${ }^{1}$ Optoelectronics Research Centre, University of Southampton, Southampton S017 1BJ, UK. ${ }^{2}$ Bell Labs, Alcatel-Lucent, Holmdel, New Jersey 07733, USA

3 Eblana Photonics Inc., Unit 31, Pearse Street, Dublin 2, Ireland. Correspondence and requests for materials should be addressed to Z.L.

(email: z.liu@soton.ac.uk).
} 
$\mathrm{D}$ irect modulation of a laser ${ }^{1}$ has always been used in optical telecommunications. Although distance-capacity product capabilities using this approach have been limited, it represents a very cost-effective solution, making it a prime choice especially when transmission distance is limited, for example, within a data centre or a supercomputer (hundreds of thousands within a single supercomputer) or in 'last-mile' telecommunications. For long-distance or high-capacity transmission, however, digital optical signals are generated using a continuous-wave (CW) laser source and an external Mach-Zehnder modulator ${ }^{2}$. To respond to the ever-increasing requirements on data transfer capacity, on-off-keyed signalling is gradually being replaced by quadrature amplitude modulation (QAM) signalling ${ }^{3}$ that enables increasing the capacity without the need to scale up the bandwidth of the electrical parts of the system, which is both costly and power hungry ${ }^{3}$. Nevertheless, QAM signalling requires in-phase-quadrature (IQ) modulator structure that is more complicated than on-off-keyed and places more stringent requirements on the modulator performance. Modulators based on the electro-optic effect (such as the industry-standard $\mathrm{LiNbO}_{3}$-based devices) offer superior performance relative to modulators exploiting charge-carrier effects in semiconductors, which, although capable of generating larger refractive index changes, also inherently impart undesirable amplitude modulation. Although $\mathrm{LiNbO}_{3}$-based modulators offer unparalleled performance, they are long (centimetre-scale), expensive and difficult to integrate with the laser, making the dense integration of tens to hundreds of transmitters as now required challenging. Consequently, major effort is being directed to obtain equivalent performance using other materials for the modulator including $\operatorname{InP}^{4,5}, \mathrm{GaAs}^{6}$ and silicon ${ }^{7}$. However, besides parasitic residual amplitude modulation, these modulators suffer from high propagation loss, allowing only for short devices requiring large radio frequency (RF) drive voltages. Nevertheless, through intensive research, devices generating QAM signals have recently appeared enabled both by improved modulator design and the use of digital signal processing (DSP) at both transmitter and receiver to compensate for the non-ideal phase and amplitude transfer functions. The current state of the art includes: a 40-GBaud InP modulator delivering $160 \mathrm{Gbits}^{-1}$ via external polarization multiplexing of two QPSK (quadruple phase shift keying) ${ }^{4}$ signals, an InP-based photonic integrated circuit (laser + modulator integrated) capable of 256-Gbit s ${ }^{-1}$ operation using 32-GBaud polarization-multiplexed $16 \mathrm{QAM}^{5}$; a GaAs-based modulator at $150 \mathrm{Gbits}^{-1}$ using 25-GBaud QAM signals; ${ }^{6}$ and finally a silicon-based modulator capable of $224 \mathrm{Gbits}^{-1} 16 \mathrm{QAM}^{7}$. Although the progress has been impressive, several drawbacks still remain. Specifically, the number of high-speed RF connections to the modulator is twice that ideally is needed due to the requirement for push-pull operation (increasing packaging cost and power requirements); the required RF power is high; computationally heavy DSP is needed-leading to a requirement for high effective number of bits and high bandwidth converters.

It is worth mentioning that other IQ modulator configurations have also been reported, for example, using four amplitude modulators ${ }^{8}$ and more complex configurations based on a greater number of phase modulators that relax the requirements on the $\mathrm{RF}$ drive signals ${ }^{9}$ or improve the linearity of the modulator response ${ }^{10}$.

Here we use a combination of optical injection locking (OIL) and direct modulation of semiconductor lasers to generate IQmodulated optical signals. Our approach allows for all the benefits of direct modulation, including high linearity, reduced power consumption, small footprint and ease of integration, and opens up a promising new route to the realization of cost-effective, high-performance monolithically integrated QAM transmitters. However, the impact of this work can be seen far beyond telecommunications. Directly current modulated lasers are devices of huge commercial relevance and are widely used in many applications, for example, optical sensing and high-power fibre lasers. In these fields, the inability to accurately control the full optical field through direct current modulation is a fundamental problem, limiting applications to just those requiring intensity modulation and constraining broader use. The new capability we demonstrate here could have a significant impact within the many scientific and engineering communities that are directly concerned with or exploit laser radiation. For example, the coherent combination of high-power lasers could greatly benefit from the control of amplitude and phase that we have demonstrated from low-cost semiconductor devices and may open the way to significantly higher powers and useful functionalities (for example, beam steering), which require precise control of the phase properties of the combined beams. Another example lies in the photonic generation of arbitrary $\mathrm{THz}$ signals as needed in a wide variety of emerging ultrafast applications. A final example is laser radar where complex beam modulation is used to provide high-resolution ranging information.

\section{Results}

Principle of our IQ transmitter. Schematics of our method ${ }^{11-13}$ showing how to build QAM signals from directly amplitudemodulated (DM) lasers are sketched in Fig. 1a for two specific exemplar signals (QPSK and 16QAM), although we emphasize that the approach can readily be adapted to work for arbitrary IQ modulation. First, we start with amplitude modulation (two levels for QPSK and four levels for 16QAM; Fig. 1a). By combining two such signals with a $90^{\circ}$ relative phase shift, we obtain QPSK and 16QAM constellations, albeit not centred at zero (due to the residual carrier). We remove the carrier via destructive interference with an unmodulated component of the carrier (Fig. 1a), finally obtaining ideal QPSK and 16QAM signals.

There are two main obstacles that need to be overcome to realize a DM laser-based 'IQ Transmitter' based on the principle shown in Fig. 1a. First, a directly modulated semiconductor laser produces a large frequency chirp ${ }^{14}$ that can be represented as circles rather than points in a constellation diagram (Fig. 1b). The second obstacle in realizing the concept is that the two independent lasers carrying the independent data streams must be coherently combined (with $90^{\circ}$ phase shift in our case, Fig. 1a) and thus must be mutually coherent.

We have addressed both issues using an effect known as $\mathrm{OIL}^{15,16}$. In OIL, a signal from a 'Master' laser (in our case a CW laser) is injected into the cavity of a 'Slave' laser (a directly modulated laser in our case; Fig. 1a). Under certain conditions, the slave phase locks to the master, even when directly modulated. The bandwidth (that is, difference in frequency of the free-running lasers) over which this locking occurs is called the OIL bandwidth ${ }^{17}$. Once the directly modulated slave is locked to a CW master, its chirp is suppressed (Fig. 1b) $)^{18,19}$ and its modulation bandwidth can be significantly increased (for example, up to $80 \mathrm{GHz}^{20}$ ). Moreover, using the same master for two slave lasers allows mutual coherence between the three devices to be established and hence for the stable coherent superposition (interference) of their outputs. This mutual coherence is critical in our transmitter-it allows us to combine the two directly modulated slaves with a $90^{\circ}$ shift, as well as to provide carrier suppression through destructive interference with a component of the master signal. 
a
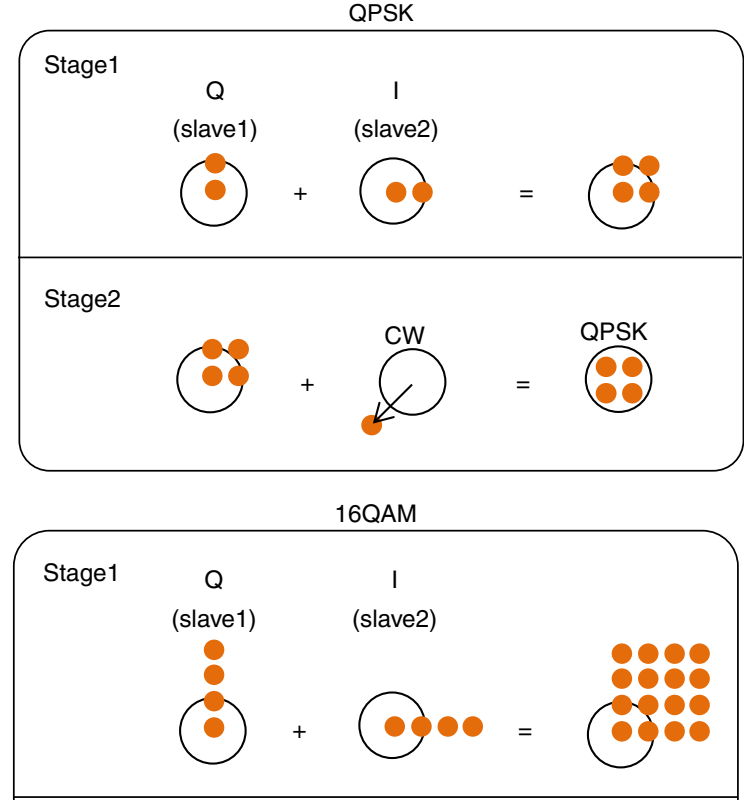

Stage2
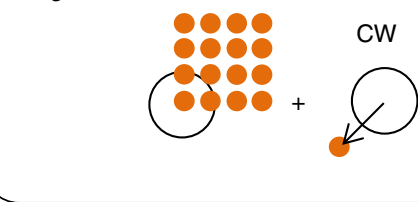

b

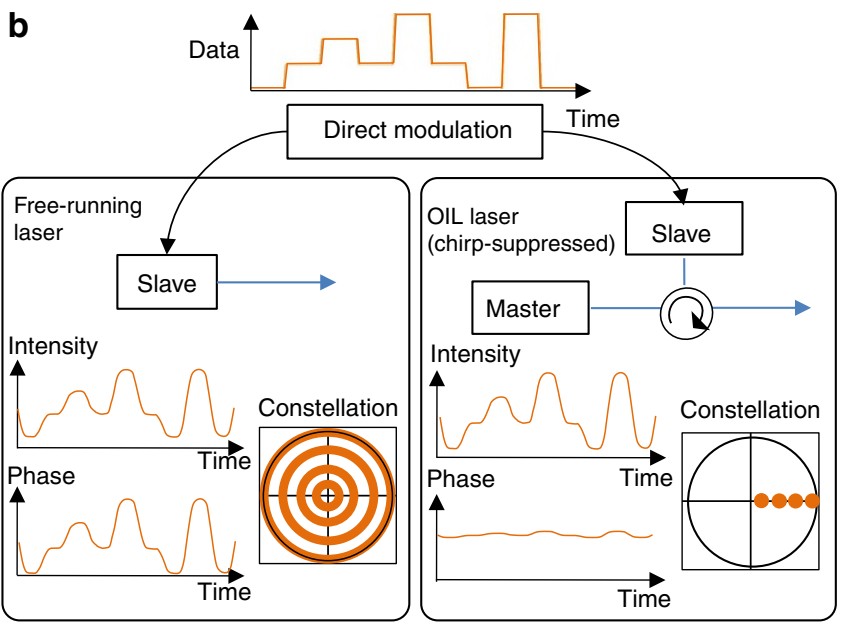

Figure 1 | Proposed transmitter based on directly modulated lasers.

(a) Principle of operation of our transmitter shown using constellation diagrams (in the complex plane) to generate QPSK and 16-level QAM (16QAM) signals. It uses amplitude modulation as the basic building block. Two amplitude-modulated signals are combined (Stage 1), making a signal with a constellation similar to that desired. By removing the carrier component of the signal via interference with the master signal (Stage 2), the desired modulation format signal is obtained. This method allows for an arbitrary level of carrier signal to be present at the output, which is difficult to achieve with a traditional IQ modulator without inducing signal distortion. (b) A sketch explaining the difference between a free-running and OIL assisted directly modulated semiconductor laser. Due to the large chirp associated with the directly modulated laser, the constellation consists of four rings rather than four points, making it unsuitable for IQ modulation. However, OIL causes the four circles to 'collapse' into four distinct points in the IQ plane.
Experiment. The proof-of-principle set-up for our transmitter is shown in Fig. 2 (described in the Methods section). First, we investigated wavelength tunability. Figure $3 \mathrm{a}$ shows the emitted spectra of a free-running slave for three different temperature settings, showing that it can emit light across almost the entire C-band. Under OIL, the laser becomes single mode as shown in Fig. $3 b$ for the three different wavelengths and three slave temperature settings shown in Fig. 3a. By coarse tuning of the slave temperature, OIL can be achieved across the full C-band $(1,530-1,560 \mathrm{~nm})$. Figure $3 \mathrm{c}$ shows the constellations obtained for binary phase shift keying for various bit rates and wavelengths, showing almost wavelength-independent performance-all were achieved using just one slave laser.

The results of further experiments using an arbitrary waveform generator (AWG) to generate the RF data are shown in Fig. 4a,b (single-carrier QPSK and 16QAM) and Fig. 4c,d (orthogonal frequency-division multiplexed (OFDM) with QPSK and 16QAM). Results obtained with a pseudorandom bit sequence (PRBS) used to generate QPSK at baud rates beyond the capabilities of our AWG (20 and $28 \mathrm{GBaud}$ ) are shown in Fig. 5. For direct comparison, we present results obtained with a 22.5-GBaud $\mathrm{LiNbO}_{3}$ IQ modulator (Figs 3 and 4).

Figure $4 \mathrm{a}$ shows that our transmitter has about $0.8 \mathrm{~dB}$ optical signal-to-noise (OSNR) penalty at bit error ratio (BER) of $10^{-3}$ with respect to our $\mathrm{LiNbO}_{3}$ modulator after propagation through $230 \mathrm{~km}$ of optical fibre. Figure $4 \mathrm{~b}$ shows that our transmitter outperforms $\mathrm{LiNbO}_{3}$ at high BER (above $2 \times 10^{-3}$ ), but performs worse for lower BER when the signal is propagated through $230 \mathrm{~km}$ of optical fibre. In the constellation (obtained after a dynamic equalization stage), we see a slight distortion, which is a consequence of the (weak) residual chirp present in our transmitter.

In Fig. 4c, we show that our transmitter is capable of generating arbitrary modulated signals by using OFDM. As we see from the spectra (inset), our transmitter shows a linear mapping of electrical signal to optical carrier. The constellation diagrams (obtained after $230 \mathrm{~km}$ of fibre propagation and after equalization) show clear constellation clusters and that our transmitter is only slightly inferior to our $\mathrm{LiNbO}_{3}$ IQ modulator biased at $V_{\pi}$ (0.7- and 0.5-dB OSNR penalty for OFDM-QPSK and OFDM16QAM, respectively, at a BER of $10^{-3}$ ). As OFDM incorporating a component of optical carrier is useful in demodulation, IQ modulators are usually biased slightly away from $V_{\pi}$ to obtain the carrier tone ${ }^{21}$. Under the practical assumption of biasing $\mathrm{LiNbO}_{3}$ at $1.05 \mathrm{~V}_{\pi}$, our transmitter (which allows the addition of a carrier tone without waveform distortion) actually outperforms $\mathrm{LiNbO}_{3}$ for OFDM-16QAM (most probably due to the better linearity of our transmitter, which is discussed in Supplementary Figs 1 and 2), while being very close to it for OFDM-QPSK (Fig. 4d).

Further, we investigated how far in terms of baud rate we can push our transmitter. This proved to be beyond the bandwidth of our AWG, and thus we used a (faster) PRBS generator with two complementary outputs, but which restricted us to work only with QPSK. We found that the slave modulation bandwidth was about $14 \mathrm{GHz}$, allowing us to go up to $28 \mathrm{GBaud}$-results for 20 and 28 GBaud are shown in Fig. 5.

\section{Discussion}

As shown above, the performance of our transmitter approaches that of our commercial $\mathrm{LiNbO}_{3}$ IQ modulator. In addition, it requires only half the number of high-speed RF inputs with $0.8 V_{\mathrm{pp}}$ (volts peak-to-peak) drive voltage (as compared with $>2 V_{\mathrm{pp}}$ in $\mathrm{LiNbO}_{3}$, InP or GaAs and $5 V_{\mathrm{pp}}$ in silicon), thereby requiring 25 times less $\mathrm{RF}$ power. Similarly to external modulators, our transmitter can be modified to operate in a push-pull mode or using binary-only RF data streams-details are 
a
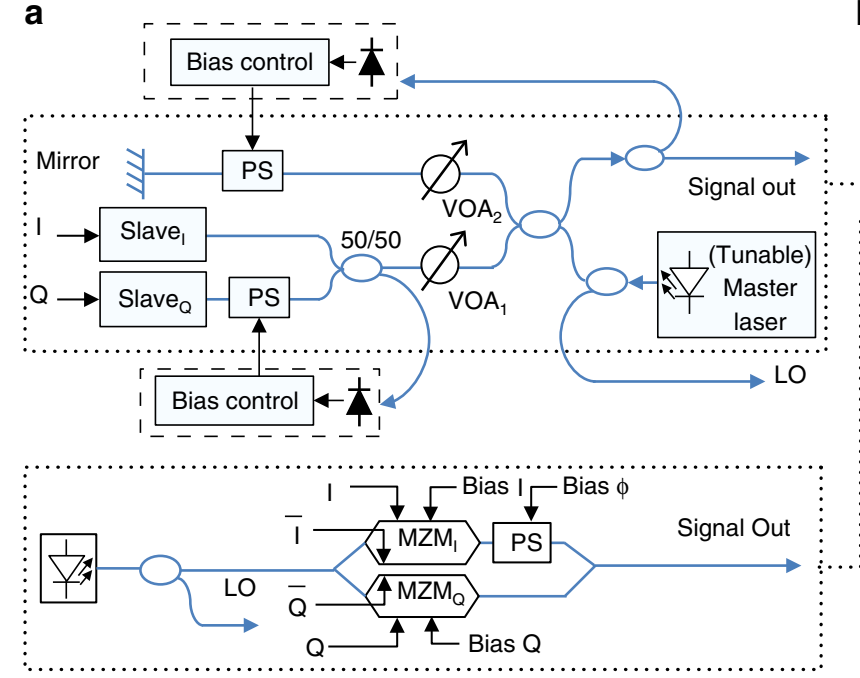

b

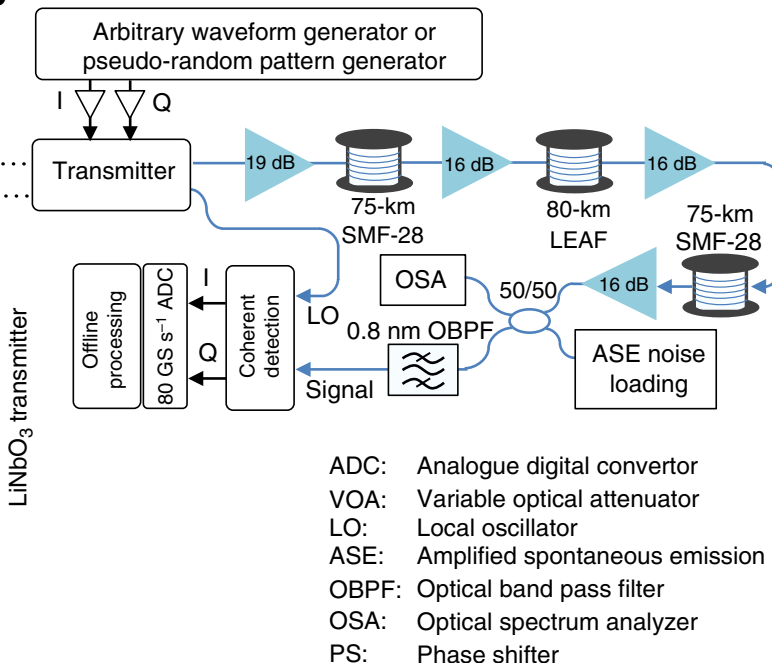

Figure 2 | Experimental implementation. (a) Experimental set-up of our transmitter system used in our proof-of-principle experiments. (a) Upper box: physical implementation of our transmitter; (a) lower box: conventional $\mathrm{LiNbO}_{3}$-based IQ transmitter used for comparative purposes. It is worth noting that our transmitter requires two times less RF data input signals compared with a standard IQ modulator-based system. Due to the good optical performance, only standard DSP was needed at the receiver side, even though the directly modulated lasers used in our demonstration are just simple Fabry-Perot semiconductor lasers and have not at all been optimized for this application. (b) Set-up of our transmission experiment.

a

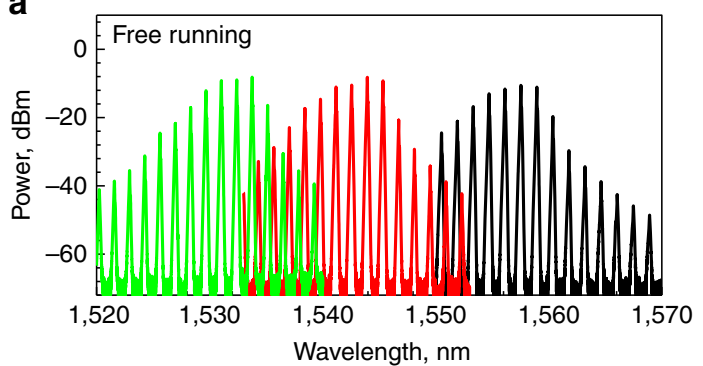

b

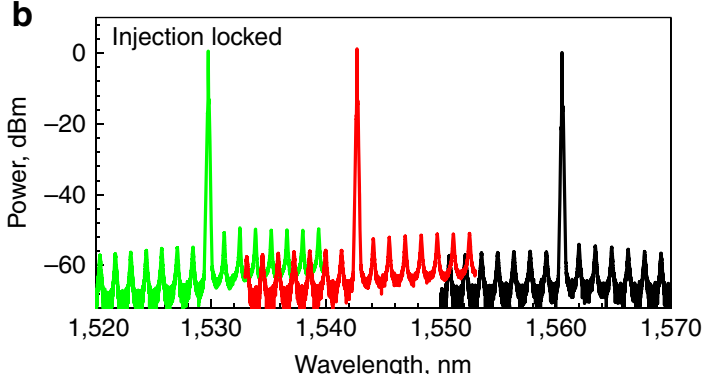

C

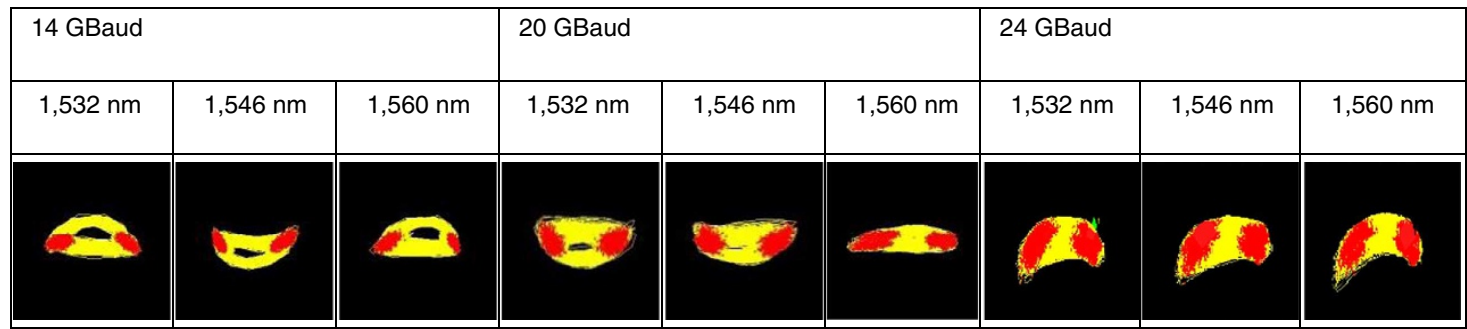

Figure 3 | Wavelength tunability of our transmitter. (a) Spectra of the free-running slave for three temperature settings. (b) Spectra of slave injection locked to a master that was tuned to 1,530 nm (green), 1,543 nm (red) and 1,561 nm (black). (c) Eye diagrams for binary phase shift keying modulation (only one slave was on and driven by a two-level data stream) generated at 1,532, 1,546 and 1,560 nm at 14, 20 and 24 GBaud, respectively.

given in Supplementary Figs 3 and 4. Although the lasers in our transmitter require temperature control, we expect that only one temperature controller would be needed if all lasers within the transmitter are integrated into a single integrated photonic circuit. As for competing technologies, they generally require temperature control as well. Although $\mathrm{LiNbO}_{3}$ modulators do not themselves require temperature control, the signalling laser usually does. Thus, we conclude that all existing/currently researched solutions require at least one temperature controller. Regarding the power consumption of the various feedback control loops, it is to be appreciated that these are of very low speed ((sub-) $\mathrm{kHz}$ speed) and hence they can be designed to consume negligible amounts of power in comparison with the temperature control and RF power consumption. With regards to energetic efficiency, our transmitter has an inherent $3-\mathrm{dB}$ loss as it exploits amplitude modulation in which half of the optical power is within the carrier and that needs subsequently to be removed. The removal process (interference with the master CW laser) requires an identical amount of power from the master laser as is contained in the carrier to be removed, resulting in $5 \mathrm{~dB}$ loss (for example, $1 \mathrm{~mW}$ of amplitude-modulated signal will become $0.5 \mathrm{~mW}$ when the carrier is removed and a further $0.5 \mathrm{~mW}$ is needed to remove the carrier. Thus, we need a total of $1.5 \mathrm{~mW}$ of optical power to obtain $0.5 \mathrm{~mW}$ of carrier-less modulated signal at the output). To obtain the same level of linearity in the modulation response of a $\mathrm{LiNbO}_{3}$ modulator as we have in our 
a

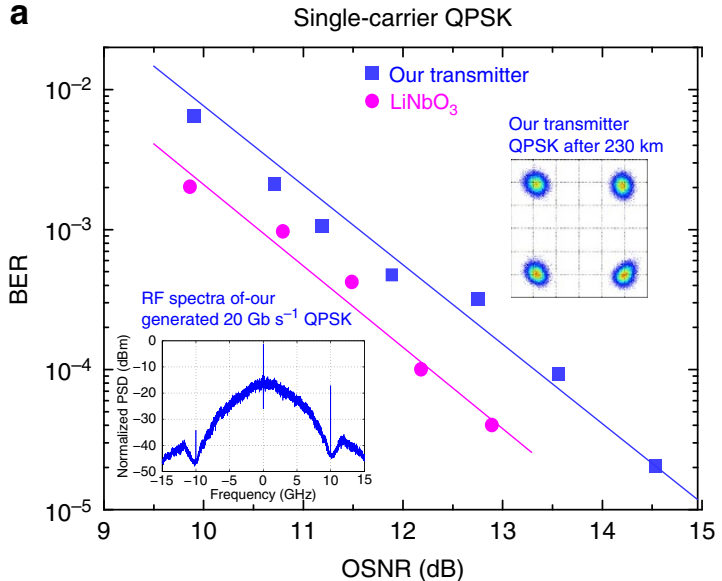

C

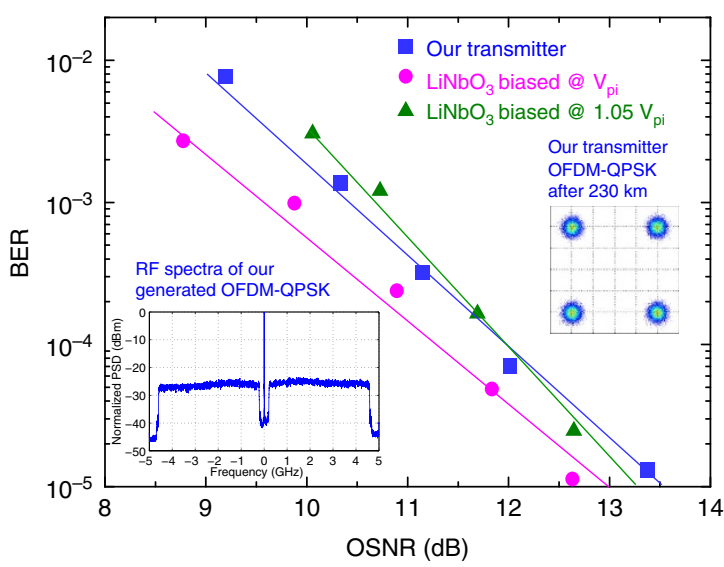

b

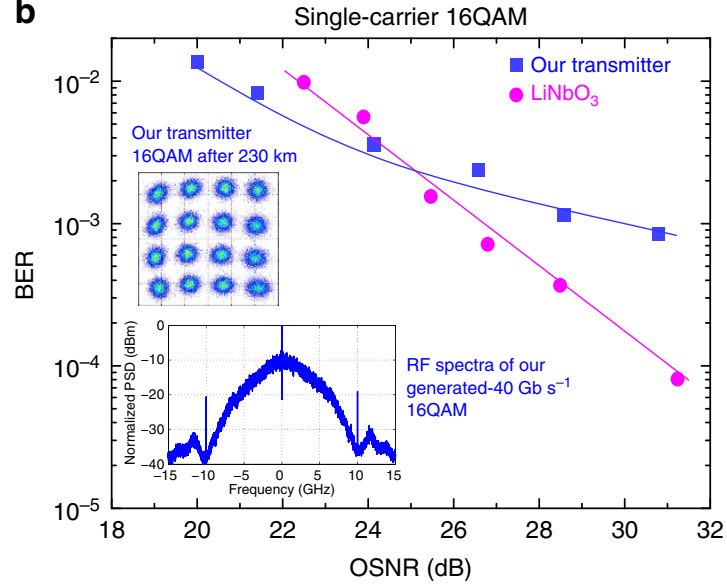

d

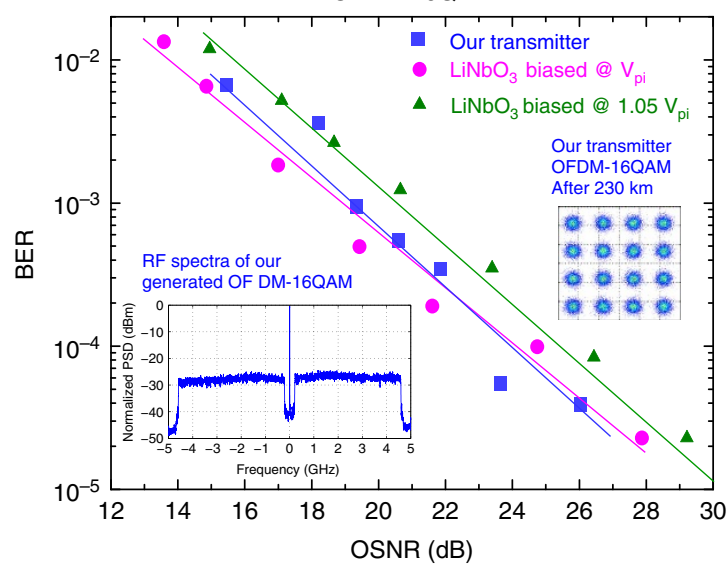

Figure 4 | Performance of our transmitter with different modulation formats. (a) Single-carrier QPSK format. (b) Single-carrier 16-level QAM (16QAM) format. (c) OFDM signal with subcarriers encoded using QPSK modulation format. (d) OFDM signal with subcarriers encoded using 16QAM modulation formats. RF data signal generated by AWG. Spectra of the generated signal and constellation after the transmission are shown as insets.

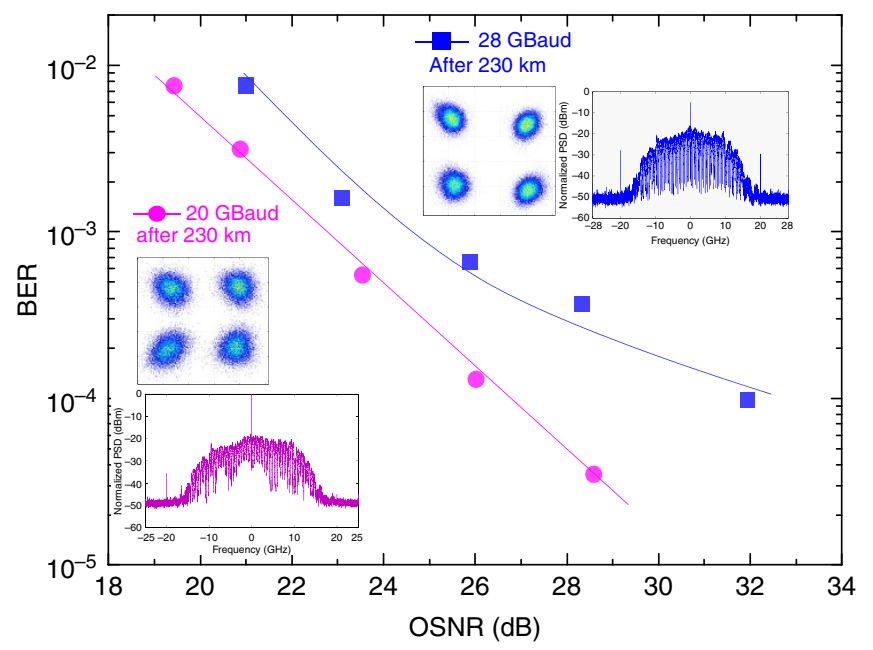

Figure 5 | Performance of our transmitter at high baud rates. Singlecarrier QPSK data are generated with PRBS RF generator. Spectra of the generated signal and constellation after the transmission are shown as insets. Blue squares: 28 Gbaud; violet circles: 20 Gbaud.

transmitter, the $\mathrm{LiNbO}_{3}$ modulator needs to be operated within its linear response regime only, increasing its loss (typically $2-5 \mathrm{~dB}$ ) by another $3 \mathrm{~dB}$, making our approach comparable in terms of energetic efficiency to a $\mathrm{LiNbO}_{3}$ IQ modulator. In addition, electrical power required to generate the sacrificed carrier power should be significantly smaller than the savings in the high-speed RF power, as our transmitter requires only half of the RF streams.

Although the data rates demonstrated in our current proof-ofprinciple experiments are relatively modest compared with the most recent results obtained with external IQ modulators, we did so without any optimization of our transmitter components and using only off-the-shelf components designed for other applications. We expect significant improvements when using optimized slave lasers, especially when these are integrated onto a single photonic chip (for example, InP) as we envisage doing. However, even with the present transmitter, we required 25 times lower RF drive power, helped by the fact that we need only half as many RF signals, and obtain a performance reasonably close to the best state-of-the-art modulators. We consider that this makes our transmitter an ideal candidate for Metro and Access networks, where power consumption is the primary concern.

\section{Methods}

Our transmitter. The slave lasers were a pair of $250-\mu \mathrm{m}$ long, 1,550-nm AlGaInAs Fabry-Perot lasers' diode designed for operation at $2.5 \mathrm{Gbits}^{-1}$, packaged in a commercial high-speed butterfly module with thermo-electric control and an electrical connection bandwidth of $18 \mathrm{GHz}$. The laser output was directly coupled into a polarization-maintaining (PM) single-mode fibre without any in-built isolator (as needed to allow OIL). The master was either a $40-\mathrm{mW} \mathrm{CW}$ telecom-grade C-band tunable laser (used in experiments requiring tunability) or a $10-\mathrm{kHz}$ linewidth fibre laser emitting $20 \mathrm{~mW}$ of CW power at $1,555.7 \mathrm{~nm}$. Variable optical 
attenuator VOA1 attenuated the master laser signal by $3 \mathrm{~dB}$ to obtain the optimal injection power of $2 \mathrm{dBm}$. In an optimized design, VOA1 could be eliminated, thereby requiring less power from the master laser and-at the same timeallowing higher output signal power, as the slave laser output would also not need to pass through this attenuator. The free-running slave laser wavelengths were set (via temperature control) to be slightly higher than the master laser wavelength. The wavelengths were fine-tuned to obtain optimum modulation linearity and minimum residual modulation chirp ${ }^{22}$. The outputs from the two slave lasers were orthogonally combined by setting and maintaining a relative phase shift between their carriers of $90^{\circ}$ (that is, operated in quadrature). The relative phase shift between the two slave laser carriers was controlled via a piezoelectric (PZT)-driven fibre stretcher (6-cm long) placed at the output of one of the slave lasers (Fig. 2b). The feedback to keep the two slave lasers in quadrature consisted of a slow photodiode (Fig. 2b) followed by a $1-\mathrm{kHz}$ bandpass filter and a proportionalintegral (PI) loop controller. Constructive/destructive interference of the two slaves (corresponding to a relative phase of $0^{\circ}$ and $180^{\circ}$, respectively) produced maximum/minimum signal at the photodiode. Hence, in quadrature $\left(90^{\circ}\right)$, the signal at the output of the photodiode changed monotonically with relative phase between the two slave lasers and thus could be used directly as the error signal at the PI controller input. Once the two slave lasers were combined in quadrature, the carrier part of their combination was removed by destructively interfering it with a component of the master laser signal obtained via reflection from a mirror. To keep the destructive interference, another feedback loop was necessary. Again, a slow photodiode followed by a 1-kHz filter, PI loop filter and a PZT stretcher was used. However, unlike in the previous feedback loop, we needed to obtain the phase lock at the minimum of the signal coming out of the slow photodiode (as we operate at the interference minimum). We introduced a small dither signal at the PZT fibre stretcher $\left(15 \mathrm{kHz}\right.$, producing $<1^{\circ}$ phase dither), which, in conjunction with lock-in detection, allowed for a suitable error signal. In our experiments, the OIL conditions (injected power, frequency detuning between the free-running master and slave lasers) were set for optimum performance. In practice, these could be maintained by implementing further simple, low-bandwidth phase-lock loops, as we did previously for slave lasers under CW operation ${ }^{23}$. The transmitter set-up is made entirely of PM fibres and PM-coupled components to avoid any polarization drift. Further, to maintain mutual coherence between the three interfering signals (two slaves and a portion of the master), the difference in paths travelled by these three signals was kept to $<20 \mathrm{~cm}$, being well below the coherence length of the master laser. Finally, to minimize phase drifts due to environmental factors (temperature, acoustic pick-up), we kept the fibres carrying these signals as physically close together as possible. As a result, feedback with only sub-kHz loop bandwidth was needed to stabilize the path difference as applied via two PZT fibre stretchers. The slave lasers were biased at $43 \mathrm{~mA}$ and modulated by electrical signals generated either using a dual-channel AWG, Tektronix AWG7122C with a bandwidth of $9.6 \mathrm{GHz}$ and sampling rate of $10 \mathrm{GS} / \mathrm{s}$ or a dual-output PRBS generator capable of operating up to 56 GBaud with a 1-bit output (two levelsOFF and ON). The two RF data streams (two AWG outputs (used as I and Q) or two complementary outputs of a PRBS generator properly delayed to be decorrelated) were amplified by two RF amplifiers to $0.8 V_{\mathrm{pp}}$ and used to directly drive the two slave lasers. The RF signals sent to the two slave lasers were synchronized using a manual RF phase shifter delay line when the PRBS generator was used, or directly using the AWG. The AWG was used for the generation of the OFDM signals (QPSK and 16QAM), 10-GBaud QPSK and 16QAM (data shown in Figs 3 and 4 ). When baud rates beyond the AWG capabilities (10 GBaud) were necessary (Figs 2 and 5), the PRBS generator was used, which allowed only for the generation of QPSK signals (having just 1-bit outputs).

Digital signal processing. In the OFDM experiment, the baseband waveform samples were calculated offline based on a PRBS of $2^{17}-1$. Both QPSK and 16QAM constellation mapping were employed. An inverse Fourier transform size of 256 was used. Of the 256 subcarriers, 22 high-frequency and 11 low-frequency subcarriers are set to zero, which is common in OFDM as low-frequency channels suffer from a frequency offset penalty and high-frequency channels suffer from the effects of filtering. Eight-sample cyclic prefix is placed before and after each OFDM symbol, resulting in symbol rate of about $36.8 \mathrm{MSymb} \mathrm{s}^{-1}$ for each subcarrier. The nominal data rate for QPSK and 16QAM mapping were 16.5 and $32.9 \mathrm{Gbits}^{-1}$, respectively. The optical carrier is not fully cancelled to generate OFDM with a carrier tone ${ }^{21}$, which results in a carrier-signal ratio of $-7 \mathrm{~dB}$. A $16 \%$ training overhead was used for symbol synchronization and channel estimation. After 1-tap equalization, the BER was calculated.

In the 10-GBaud single-carrier QPSK and 16QAM experiments, the drive waveforms are differentially encoded using a quadrant rotation algorithm ${ }^{24}$ based on a PRBS of $\left(2^{19}-1\right)$. At the receiver side, we first compensated for the imperfections in the optical front end (deskew, d.c. block, power imbalance correction and IQ orthogonalization). Then, we downsampled the acquired waveforms to two samples/symbol and compensate the chromatic dispersion using a static equalizer ${ }^{25}$. After that, we used a standard 17-tap constant modulus algorithm (CMA ${ }^{25}$ and a 22 -tap multi-ring $\mathrm{CMA}^{26}$ to adaptively compensate the residual impairments for QPSK and 16QAM, respectively. As homodyne detection was used to simplify the experiment, frequency offset compensation was not needed. In the last stage, we compensated the phase noise using a feed-forward carrier recovery algorithm ${ }^{24}$. Finally, the signals were differentially decoded before BER calculation.

For the 20-GBaud and 28-GBaud QPSK experiments, we split the output of a high-speed pseudorandom pattern generator (SHF 12100B) into two branches and introduced a delay to one branch for driving I and Q (PRBS of $2^{15}-1$ ). The receiver side processing was similar to that used in the 10-GBaud experiment, except that a 13-tap sliding filter was used to compensate the phase noise $\mathrm{s}^{27}$.

In the wavelength tunability experiment (Fig. 3), homodyne detection was performed with the help of commercial Agilent DSP algorithms in-built in the Coherent receiver (Agilent DSO-X 93204A + N4391A).

Transmission link set-up. The $230-\mathrm{km}$ transmission link incorporated three spans of fibre: $75 \mathrm{~km}$ of standard single-mode fibre (SSMF-28) in the first and third span and $80 \mathrm{~km}$ of large effective area fibre in the second span. We launched $-1 \mathrm{dBm}$ of average power to avoid nonlinearities. At the receiver side, ASE-generated noise loading was used to adjust the OSNR. The OSNR was calculated from the measured signal power and noise floor at $0.1-\mathrm{nm}$ resolution. A $0.8-\mathrm{nm}$ optical bandpass filter was used to filter out the out-of-band noise. A component of CW light $(6 \mathrm{dBm})$ was tapped from the master laser and used as a local oscillator for single-polarization homodyne reception. The polarization of the received signal was manually aligned with that of the local oscillator at the receiver. After coherent detection, the electrical signal was then sampled by a $32-\mathrm{GHz}, 80-\mathrm{GS} \mathrm{s}^{-1}$ real-time oscilloscope before offline processing.

Most experiments conducted with our transmitter were repeated using a 22.5-GBaud IQ modulator from Fujitsu (FTM7960EX) for comparison purposes. To ensure optimum linearity, $V_{\mathrm{pp}}$ of the driving RF signals was reduced to $\sim 0.6 V_{\pi}$.

\section{References}

1. Harris, S. E., McMurtry, B. J. \& Siegman, A. E. Modulation and direct demodulation of coherent and incoherent light at a microwave frequency. Appl. Phys. Lett. 1, 37-39 (1962).

2. Kumar, S. \& Deen, M. J. Fiber Optic Communications: Fundamentals and Applications (Wiley, 2014).

3. Seimetz, M. High-Order Modulation for Optical Fiber Transmission (Springer, 2009).

4. Prosyk, P. et al. in Proceedings of the 37th European Conference on Optical Communications (ECOC 2011), Geneva, Switzerland, Paper Th.13.A.5 (2011).

5. Chandrasekhar, S., Liu, X., Winzer, P. J., Simsarian, J. E. \& Griffin, R. A. Compact all-InP laser-vector-modulator for generation and transmission of 100-Gb/s PDM-QPSK and 200-Gb/s PDM-16-QAM. J. Lightwave Technol. 32, 736-742 (2014).

6. Schindler, P. C. et al. Monolithic GaAs electro-optic IQ modulator demonstrated at $150 \mathrm{Gbit} / \mathrm{s}$ with 64QAM. J. Lightwave Technol. 32, 760-765 (2014).

7. Dong, P. et al. Monolithic silicon photonic integrated circuits for compact $100+\mathrm{Gb} / \mathrm{s}$ coherent optical receivers and transmitters. IEEE J. Sel. Top. Quant. Electron. 6100108 (2014).

8. Kang, I. et al. A hybrid electroabsorption modulator device for generation of high spectral-efficiency optical modulation formats. Opt. Express 16, 8480-8486 (2008).

9. Chiba, A. et al. 16-level QAM by monolithic quad-parallel Mach-Zehnder optical modualator. Elctron. Lett. 46, 220-221 (2010).

10. Yamazaki, H. et al. in Proceedings of the Optical Fiber Communication Conference (OFC 2013), Anaheim, CA, Paper OM3C (2013).

11. Slavík, R. et al. in Proceedings of the Optical Fiber Communications Conference (OFC), Anaheim, CA, Paper JTh2A.32 (2013).

12. Slavík, R., Kakande, J., Phelan, R., O’Carroll, J. \& Richardson, D. J. in Proceedings of the Optical Fiber Communications Conference (OFC), Anaheim, CA, Paper PDP5B.5 (2013).

13. Slavík, R., Kelly, B., Phelan, R., O'Carroll, J. \& Richardson, D. J. in Proceedings of the 39th European Conference on Optical Communications (ECOC 2013), Paper P.3.22 (2010).

14. Hakki, B. W. Evaluation of transmission characteristics of chirped DFB lasers in dispersive optical fiber. J. Lightwave Technol. 10, 964-970 (1992).

15. Siegman, A. E. Lasers. Chapter 29 (University Science Books, 1986).

16. Lang, R. Injection locking properties of a semiconductor laser. IEEE J. Quant. Electron. 18, 976-983 (1982).

17. Petitbon, I., Gallion, P., Debarge, G. \& Chabran, C. Locking bandwidth and relaxation oscillations of an injection-locked semiconductor laser. IEEE $J$. Quant. Electron. 24, 148-154 (1988).

18. Mohrdiek, S., Burkhard, H. \& Walter, H. Chirp reduction of directly modulated semiconductor lasers at $10 \mathrm{~Gb} / \mathrm{s}$ by strong $\mathrm{CW}$ light injection. J. Lightwave Technol. 12, 418-424 (1994).

19. Mogensen, F., Olesen, H. \& Jacobsen, G. Locking conditions and stability properties for a semiconductor lasers with external light injection. IEEE J. Quant. Electron. QE-21, 784-793 (1985).

20. Lau, E. K. et al. Strong optical injection-locked semiconductor laser demonstrating $>100-\mathrm{GHz}$ resonance frequencies and $80-\mathrm{GHz}$ intrinsic bandwidths. Opt. Express 16, 6609-6618 (2008). 
21. Jansen, S. L., Morita, I., Schenk, T. C. W., Takeda, N. \& Tanaka, H. Coherent optical 25.8-Gb/s OFDM transmission over 4160-km SSMF. J. Lightwave Technol. 26, 6-15 (2008).

22. Murakami, A., Kawashima, K. \& Atsuki, K. Cavity resonance shift and bandwidth enhancement in semiconductor lasers with strong light injection. IEEE J. Quant. Electron. 39, 1196-1204 (2003).

23. Wu, D. S., Slavík, R., Marra, G. \& Richardson, D. J. Direct selection and amplification of individual narrowly spaced optical comb modes via injection locking: design and characterization. J. Lightwave Technol. 31, 2287-2295 (2013).

24. Pfau, T., Hoffmann, S. \& Noé, R. Hardware-efficient coherent digital receiver concept with feedforward carrier recovery for M-QAM constellations. J. Lightwave Technol. 27, 989-999 (2009).

25. Savory, S. J. Digital coherent optical receivers: algorithms and subsystems. IEEE J. Sel. Top. Quant. Electron. 16, 1164-1179 (2010)

26. Winzer, P. J., Gnauck, A. H., Doerr, C. R., Magarini, M. \& Buhl, L. L. Spectrally efficient long-haul optical networking using $112-\mathrm{Gb} / \mathrm{s}$ polarization-multiplexed 16-QAM. J. Lightwave Technol. 28, 547-556 (2010).

27. Viterbi, A. J. \& Viterbi, A. M. Nonlinear estimation of PSK-modulated carrier phase with application to burst digital transmission. IEEE Trans. Inf. Theory 29, 543-551 (1983).

\section{Acknowledgements}

This research has received funding from EPSRC Fellowship grant agreement no. EP/K003038/1 and EPSRC grant EP/I061196X (Photonic Hyperhighway)

\section{Author contributions}

Z.L. designed and carried out all the experiments involving advanced modulation formats. R.S. and J.K. conceived the idea. R.S. built the transmitter and carried out preliminary tests. R.S. led the work. D.J.R. provided overall guidance and support. Z.L., J.K., R.S. and D.J.R. wrote the manuscript. B.K., J.O. and R.P. designed and manufactured the semiconductor slave lasers.

\section{Additional information}

Supplementary Information accompanies this paper at http://www.nature.com/ naturecommunications

Competing financial interests: The authors declare no competing financial interests.

Reprints and permission information is available online at http://npg.nature.com/ reprintsandpermissions/

How to cite this article: Liu, Z. et al. Modulator-free quadrature amplitude modulation signal synthesis. Nat. Commun. 5:5911 doi: 10.1038/ncomms6911 (2014).

(c) (i) This work is licensed under a Creative Commons Attribution 4.0 International License. The images or other third party material in this article are included in the article's Creative Commons license, unless indicated otherwise in the credit line; if the material is not included under the Creative Commons license, users will need to obtain permission from the license holder to reproduce the material. To view a copy of this license, visit http://creativecommons.org/licenses/by/4.0/ 